\begin{abstract}
Large-scale efforts are underway to map the neuronal connectomes of many animals, from flies to humans. Remarkably, first complete reconstruction of an animal connectome was reported more than 30 years ago, for the nervous system of the nematode $C$. elegans. Through largely manual reconstruction of serial electron micrographs, White et al characterized the morphology of each of the 302 neurons of the adult hermaphrodite worm and mapped the chemical and electrical synapses that connect them. The availability of this wiring diagram has had an important impact on behavioral neurobiology and network science, and offers a guide to the future impact of connectomics on neuroscience.
\end{abstract}

Between 1996 and 2003, the genome sequences of the yeast, worm, fly and human were completed. It became clear almost immediately that a comprehensive mapping of an organism's genetic material could provide transformative insight into its molecular and cell biology. Neuroscientists began to ask whether a complete mapping of synaptic connections in the brain--a so-called connectome--could provide similar insight into the nature of mental phenomena and the mechanistic basis of behavior[1]. By 2010, the Human Connectome Project had begun, with serious efforts also underway to map the neuronal connectomes of the smaller brains from the mouse and fruit fly.

However, the first complete neuronal connectome, of the nematode $C$. elegans, had already been completed, a quarter-century ahead of schedule. In 1986, John White and his colleagues at the MRC Laboratory of Molecular Biology in Cambridge published a complete wiring diagram for the $C$. elegans nervous system, in a monograph [2] (running head: 'The Mind of a Worm') that filled up an oversized issue of the Philosophical Transactions of the Royal Society. In this paper, described the EM-level structure of each neuron of the adult hermaphrodite, including their axonal and dendritic morphologies and all their pre- and post-synaptic connections. The resulting connectome consisted of 302 identified neurons, connected by approximately 10,000 chemical synapses and 1000 gap junctions. Apart from the wiring diagram of the C. elegans male, published in 2009 [3], it remains the only complete neuronal connectome published to date.

The effort to map the $C$. elegans neuronal connectome had its origins in the 1960s, when Sydney Brenner chose $C$. elegans as an experimental organism. Believing that the fundamental questions of molecular biology were well on their way to being solved, Brenner was looking for a way to investigate what he considered the most interesting biological mysteries: multicellular development and the mechanisms of nervous system function. Free-living nematodes like $C$. elegans offered two major advantages (first noted by [4]) for addressing these questions: its rapid reproduction, hermaphroditic mating system, and small genome size made it ideal for genetic analysis, and its small and reproducible cell number made it possible, at least in theory, to determine its anatomy completely and precisely. $C$. elegans specimens in particular proved amenable to serial electron microscopy, a key requirement for mapping the synapse-level structure of the nervous system. Indeed, serial electron microscopy was successfully used to reconstruct portions of the $C$. elegans nervous system in the 1970s: Donna Albertson and Nicol Thomson[5] reconstructed the anatomy of the 68-cell pharynx (including its 20 pharyngeal neurons) and White and 
colleagues reconstructed the connectivity of the ventral nerve cord[6]. The next step was to apply this approach to the rest of the nervous system.

Modern connectomics relies on several recent technological innovations that make it possible to trace the processes of individual neurons through densely-packed samples of neuropil and identify the individual synapses that connect them. In particular, focused ion beam-scanning electron microscopy (FIB-SEM) has greatly facilitated the collection of volume image data, with reliably undistorted, synapse-level resolution in all three dimensions[7], and sophisticated tools have been developed for computer-assisted tracing and reconstruction of neural structures from these image data[8]. As neither of these technologies existed in the 1970s, the reconstruction of the worm connectome relied on the exceptional skills of two technicians: Nicol Thomson, who meticulously generated series of hundreds of serial transmission EM sections, and Elaine Southgate, who traced individual neuronal processes through these slices using a marker pen. More than a decade of heroic work was required to hand-map relatively small connectome of $C$. elegans. It was a monumental achievement, carried out well before the appropriate tools for the job existed.

The arrival of the worm connectome was a milestone that established $C$. elegans as an experimental organism for behavioral neuroscience. Since $C$. elegans neurons are tiny and relatively inaccessible, traditional electrophysiological approaches for studying neural circuits in the worm have always been difficult. The availability of a complete connectome map made it possible to investigate the neural basis of behavior using an alternative approach: mechanistic hypotheses could be formulated based on structural connectivity, which could then be tested by cell-specific ablation experiments complemented by genetic analysis of behavioral mutants. For example, using laser ablations guided by the connectivity map, Martin Chalfie and colleagues identified a putative circuit, consisting of mechanosensory neurons, premotor interneurons, and ventral cord motorneurons, responsible for the escape response to body touch[9]. Critical to this circuit was a set of premotor interneurons that control the direction of locomotion, 6 promoting forward crawling and 4 promoting backward crawling. This work opened up the touch circuit to subsequent genetic and molecular studies of mechanosensory mechanisms.

Subsequent analysis of connectome topology has revealed deeper insight into the functions of these neurons, particularly the premotor interneurons. Graph theoretical analysis indicated that these neurons represent highly interconnected hub nodes within the broader connectome, with the potential to broadly integrate activity across the nervous system[10]. This hypothesis has been supported by recent imaging studies showing that ascending connections from the premotor network modulates the reliability of descending sensory information from the olfactory circuit[11]. Moreover, whole-brain imaging studies carried out at single-neuro resolution indicate that the premotor interneurons are important drivers can of global brain states[12]. Thus, knowledge of connectome structure has continued to enable the generation of testable hypotheses about specific as well general roles of individual neurons.

The $C$. elegans connectome has also been influential in the developing field of network science. Indeed, as the only complete neural network mapped at the cellular level, the worm connectome has provided real-world validation of several network concepts subsequently 
seen to be widespread in other biological networks. For example, the worm connectome was the first biological example of a small-world network, exhibiting the properties of higher-than-expected clustering and shorter-than-expected path length[13]; such smallworldness has subsequently been observed in larger neural systems. The $C$. elegans nervous system also contains a core of interconnected hub neurons, or rich club[10], a phenomenon seen on a macro scale in human cerebral cortex. Finally, the $C$. elegans connectome contains a number of overrepresented motifs, or microcircuits, including various permutations of the feed-forward circuit[14]. Many of these motifs also appear to function as building blocks of the neuronal circuitry in bigger brains.

For many years, the $C$. elegans connectome has been unique, but soon neuronal connectomes will be available for other organisms. The recent history of $C$. elegans research provides a glimpse not only into the power of connectomics, but also into its limitations. More than 30 years after the publication of White et al., and despite intensive experimentation in the intervening decades, our understanding of how even a 302-neuron connectome generates behavior is far from complete. This highlights a crucial difference between the genome and the connectome: whereas knowledge of the genetic code makes it straightforward to infer the functions of proteins encoded by genomic sequences, there are no comparable theories that allow us to clearly infer functionality from neuronal connectivity patterns. Clearly, a better understanding of how network topology relates to neural circuit function will be needed if we are to make sense of larger neuronal connectomes [15]. The $C$. elegans neuronal connectome provides a fertile ground to develop such theories and to test them experimentally.

1. Lichtman, J.W. and Sanes, J.R. (2008) Ome sweet ome: what can the genome tell us about the connectome? Curr Opin Neurobiol 18 (3), 346-53.

2. White, J. et al. (1986) The structure of the nervous system of the nematode Caenorhabditis elegans Phil. Trans. R. Soc. Lond. (Biol.) 314, 1-340.

3. Jarrell, T.A. et al. (2012) The connectome of a decision-making neural network. Science 337 (6093), 437-44.

4. Dougherty, E.C. and Calhoun, H.G. (1948) Possible significance of free-living nematodes in genetic research. Nature 161 (4079), 29.

5. Albertson, D.G. and Thomson, J.N. (1976) The pharynx of Caenorhabditis elegans. Philos. Trans. R. Soc. Lond. B Biol. Sci. 275, 299-325.

6. White, J.G. et al. (1976) The structure of the ventral nerve cord of Caenorhabditis elegans. Philos Trans R Soc Lond B Biol Sci 275 (938), 327-48.

7. Hayworth, K.J. et al. (2015) Ultrastructurally smooth thick partitioning and volume stitching for large-scale connectomics. Nat Methods 12 (4), 319-22.

8. Saalfeld, S. et al. (2009) CATMAID: collaborative annotation toolkit for massive amounts of image data. Bioinformatics 25 (15), 1984-6.

9. Chalfie, M. et al. (1985) The neural circuit for touch sensitivity in Caenorhabditis elegans. J Neurosci 5 (4), 956-64.

10. Towlson, E.K. et al. (2013) The rich club of the C. elegans neuronal connectome. J Neurosci 33 (15), 6380-7.

11. Gordus, A. et al. (2015) Feedback from network states generates variability in a probabilistic olfactory circuit. Cell 161 (2), 215-27. 
12. Kato, S. et al. (2015) Global brain dynamics embed the motor command sequence of Caenorhabditis elegans. Cell 163 (3), 656-69.

13. Watts, D.J. and Strogatz, S.H. (1998) Collective dynamics of 'small-world' networks. Nature 393 (6684), 440-2.

14. Varshney, L.R. et al. (2011) Structural properties of the Caenorhabditis elegans neuronal network. PLoS Comput Biol 7 (2), e1001066.

15. Yan, G. et al. (2017) Network control principles predict neuron function in the C. elegans connectome. Nature in press.

\section{Figure legend}

A portion of the $C$. elegans connectome. Forward (blue) and backward (orange) premotor interneurons are highlighted. Chemical synapses are indicated by arrows; bars indicate electrical synapses/gap junctions. Each neuron (e.g. AVA) is designated by a three-letter name. This figure is adapted from Figure 21 of White et al[2].

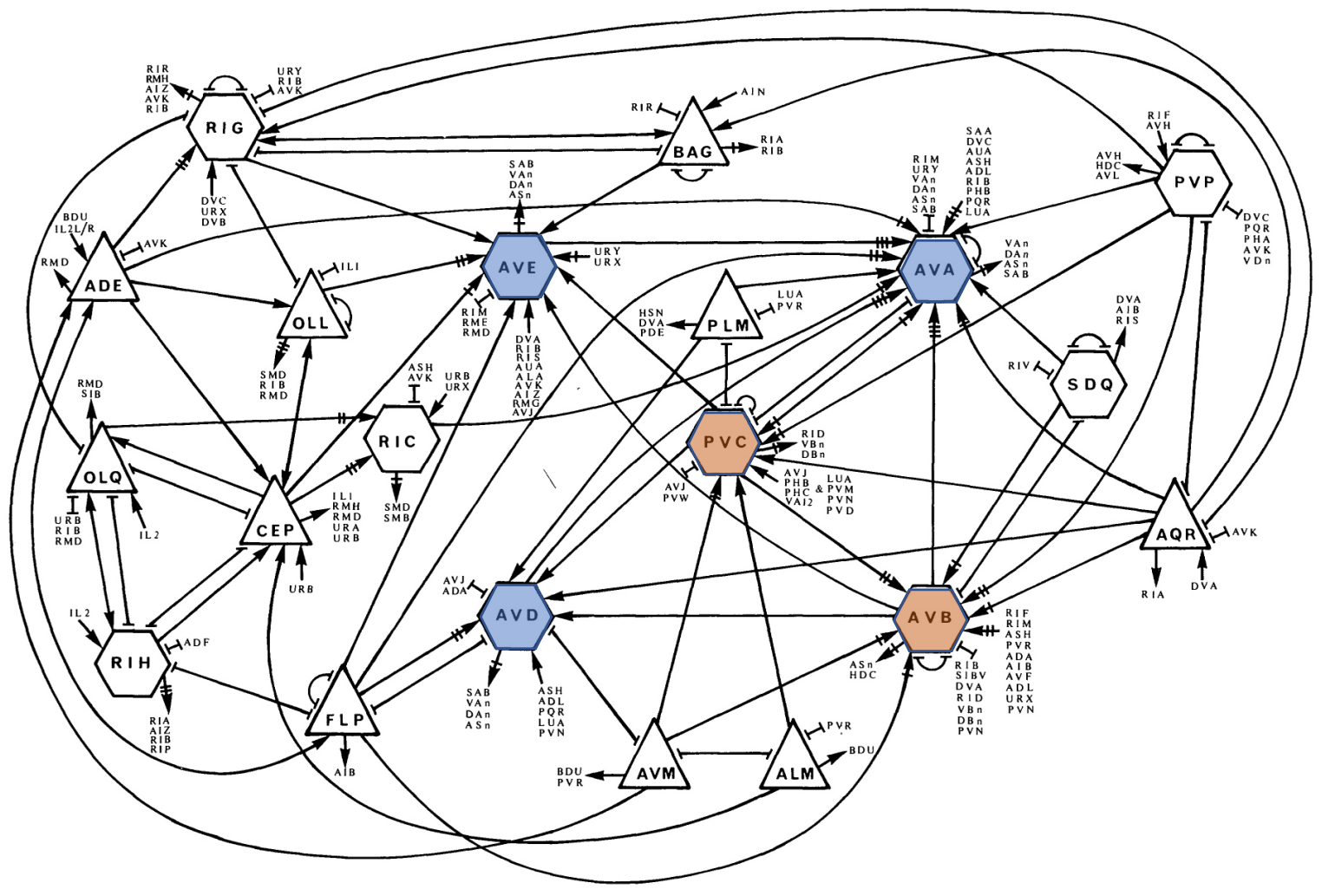

